\title{
A DIVISIBILITY PROPERTY OF BINOMIAL COEFFICIENTS VIEWED AS AN ELEMENTARY SIEVE
}

\author{
RICHARD H. HUDSON \\ Department of Mathematics \\ Computer Science, and Statistics \\ University of South Carolina \\ Columbia, South Carolina 29208 U.S.A. \\ and \\ KENNETH S. WILLIAMS \\ Department of Mathematics \\ Carleton University \\ Ottawa, Ontario, Canada K1S 5B6 \\ (Received December 2, 1981)
}

ABSTRACT. The triangular array of binomial coefficients

\begin{tabular}{l|llll}
\multicolumn{1}{c}{0} & 1 & 2 & 3 \\
\cline { 2 - 5 } 1 & 1 & & & \\
2 & 1 & 1 & & \\
3 & 1 & 2 & 1 & \\
& & 3 & 3 & 1
\end{tabular}

is said to have undergone a $j-s h i f t$ if the $r$-th row of the triangle is shifted $r j$ units to the right $(r=0,1,2, \ldots)$. Mann and Shanks have proved that in a 2shifted array a column number $c>1$ is prime if and only if every entry in the c-th column is divisible by its row number. Extensions of this result to $j$-shifted arrays where $j>2$ are considered in this paper. Moreover, an analog of the criterIon of Mann and Shanks [2] is given which is valid for arbitrary arithmetic progressions.

KEY WORDS ANO PHRASES. Array of binomial coefficients, primes in arithmetic progressions. 
1. INTRODUCTION AND SUMMARY.

We begin with the binomial coefficients arranged in the triangular array

$(1.1)$
1
11
121
1331

We say that this array has undergone a $j$-shift, $j$ an integer $\geq 2$, if for every $r \geq 0$ the $r-t h$ row of the triangle (1.1) is shifted $r j$ units to the right. Rows and columns in the shifted array are labelled as in [1]. Thus, for example, after a 2-shift (1.1) becomes

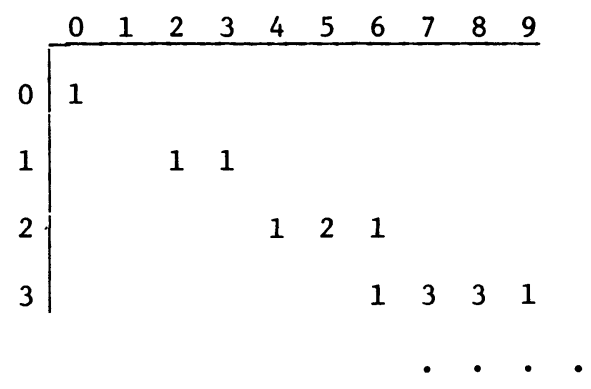

The $n+1$ binomial coefficients of $(x+y)^{n}$, namely $\left(\begin{array}{l}n \\ k\end{array}\right), k=0,1, \ldots, n$, are found in the $j$-shifted array between columns $j n$ and $(j+1) n$ inclusive. Indeed, the binomial coefficient $\left(\begin{array}{l}r \\ s\end{array}\right), 0 \leq s \leq r$, is in the $r$-th row and $(r j+s)-t h$ column (hence, we call $r$ the row number and $r j+s$ the column number of $\left(\begin{array}{l}r \\ s\end{array}\right)$. The binomial coefficients in the c-th column of a j-shifted array are given by

$$
\left(\begin{array}{c}
r \\
c-r j
\end{array}\right)
$$

where $r$ runs through the integers between $\frac{c}{j+1}$ and $\frac{c}{j}$ inclusive. For example, if $c=59$ and $j=3$, then the binomial coefficients in the 59-th column are

$$
\left(\begin{array}{l}
15 \\
14
\end{array}\right),\left(\begin{array}{l}
16 \\
11
\end{array}\right), \quad\left(\begin{array}{r}
17 \\
8
\end{array}\right), \quad\left(\begin{array}{r}
18 \\
5
\end{array}\right), \quad\left(\begin{array}{r}
19 \\
2
\end{array}\right)
$$

Henry B. Mann and Daniel Shanke [1] have proved the interesting result: 
THEOREM (Mann and Shanks). In a 2-shifted array a column number $c>1$ is prime if and only if all the binomial coefficients in the c-th column are divisible by their row number.

When $j=2$, the property that all binomial coefficients in a column be divisible by their row number is a sieving condition satisfied by the primes and only by the primes (excepting the integer 1). In Theorem 1 of this paper we show that this sieving condition is satisfied by the primes for every $j \geq 2$. The proof of Theorem 1 is a straightforward generalization of the "necessity" part of the proof of the theorem of Mann and Shanks [1].

It occurred to the first of us that although the sieving condition given above is apparently less effective when $j>2$ in that some composite numbers are allowed to slip through the sieve along with the primes, these composites may satisfy a very restrictive set of conditions or even (ideally) form a finite exceptional set beyond which the sieve catches only primes. The main result of this paper is found in Theorem 2, which deals with the converse of Theorem 1 when $j=3$. Theorem 2 asserts that if $\mathrm{n}$ is any odd integer $\neq 25$ (25 appears to be exceptional because there is no entry in the 5 -th column when $j=3$ ), then $n>1$ is prime if and only if every coefficient in the n-th column is divisible by its row number (the result is vacuously true for $n=5$ ). Thus, while superficially a 3-shift appears much less effective than a 2-shift in isolating primes (since 4, 10, 25, and 34 are allowed to slip through the sieve along with the primes), in fact the sieve is equally effective for odd integers $\neq 25$.

Criteria for even integers to be prime are presumably not of much interest since 2 is the only even prime. However, a proof that only finitely many even composites can slip through the sieve induced by a 3-shift would be of interest. (Such a proof might provide means of obtaining a result similar to Theorem 2 for $j>3$.$) At present we are only able to show (see Theorem 3$ ) that if $j=3$ and $\mathrm{n}$ is an even integer $>4$ then every coefficient in the $\mathrm{n}$-th column is divisible by its row number only if $\mathrm{n}=10,34(\bmod 96)$. In fact, the only "exceptional" integers $>4$ that we have found are 10 and 34 .

REMARK 1.1. The motivation behind the unusual primality criterion of Mann 
and Shanks arose from a study of identities of the type

$$
1-x^{j}-x^{j+1}=\prod_{n=j}^{\infty}\left(1-x^{n}\right)^{b} n
$$

see [1, p. 133]. From this viewpoint, the relevance of Theorem 1 rests in 1 ts equivalence to the assertion that for a prime $p$ each individual contribution to $x^{p}$ from a term of the form $\left(x^{j}+x^{j+1}\right)^{m} / m, j \geq 2$, must be an integer. Identities such as (1.4) have been studied by (among others) Issai Schur [3].

Finally, in section 7 , we prove an analog of the criterion of Mann and Shanks [2] that is valid for arbitrary arithmetic progressions; see Theorem 4.

\section{PROOF OF NECESSITY FOR EACH $j \geq 2$}

THEOREM 1. Let $j$ be a fixed integer $\geq 2$. Then, in a $j$-shifted array, if a column number is prime, every binomial coefficient in its column (if any) is divisible by its row number.

PROOF. We may clearly assume that $p$ is a prime column number of a nonempty column. Let $\left(\begin{array}{l}r \\ s\end{array}\right)$ be the binomial coefficient in the $r$-th row and $p$-th column, and suppose that $r \nmid\left(\begin{array}{l}\mathbf{r} \\ \mathbf{s}\end{array}\right)$. From

$$
\left(\begin{array}{c}
r-1 \\
s-1
\end{array}\right) \quad r=\left(\begin{array}{l}
r \\
s
\end{array}\right) s,
$$

we have $(r, s)>1$. Since $p=r j+s,(r, s) \mid p$, an impossibility as $p$ is prime. Thus, if the column number $p$ is prime each coefficient in the p-th column is divisible by its row number.

REMARK 2.1. From the contrapositive of Theorem 1, we see that the exhibition of a single binomial coefficient for any $j \geq 2$ that is not divisible by its row number shows that the corresponding column number is composite. For example, $\mathrm{n}=533$ is composite since the first coefficient in its column after a 20-shift is $\left(\begin{array}{l}26 \\ 13\end{array}\right)$ and this is clearly not divisible by 26 (since ps $\nmid\left(\begin{array}{c}p s \\ p\end{array}\right)$ for any prime $\mathrm{p})$. 


\section{TWO USEFUL LEMMAS}

In this section we prove two simple lemmas which we will need later.

LEMMA 1. If $\left(\begin{array}{l}a \\ b\end{array}\right)$ is an entry in the k-th column of a j-shifted array, then $\left(\begin{array}{l}a l \\ b l\end{array}\right)$ is an entry in the $k l-t h$ column of the array for any non-negative integer $l$. PROOF. Note that $k=a j+b$, so that $k \ell=(a l) j+b l$.

REMARK 3.1. The "sufficiency" part of the proof of the theorem of Mann and Shanks for odd column numbers is an immediate consequence of Lemma 1 when $j=2$. For the coefficient $\left(\begin{array}{c}\mathrm{pk} \\ \mathrm{p}\end{array}\right)$, which is not divisible by $\mathrm{pk}$, occurs in the $c=(p k(2)+p)-t h$ column precisely because $\left(\begin{array}{l}k \\ 1\end{array}\right)$ is an entry in the $(2 k+1)-t h$ column.

REMARK 3.2. If $\mathrm{j}=2$ and the column number $\mathrm{n}$ is odd and has $\mathrm{m} \geq 2$ distinct prime factors, one can produce, via Lemma 1 , $m$ coefficients in the $n$-th column that are not divisible by their row number. Thus, if $m \geq 2$, the number of binomial coefficients in an odd numbered column $n$ not divisible by their respective row numbers is at least $\mathrm{m}$. It would be interesting to improve this lower bound.

LEMMA 2. Let $c>5$ be an integer of the form $12 t+d$, where $d=1$ or 5 . The last coefficient in the c-th column of a 3-shifted array is

$$
\left(\begin{array}{c}
4 t+[d / 3] \\
d-3[d / 3]
\end{array}\right)
$$

PROOF. This follows immediately from (1.3).

\section{PROOF OF SUFFICIENCY FOR A 3-SHIFT IF $n$ IS AN ODD INTEGER $\neq 25$}

THEOREM 2. If every coefficient in the n-th column of a 3-shifted array is divisible by its row number with $n$ an odd integer not equal to 1 or 25 , then $n$ is a prime.

PROOF. Since $\mathrm{n}>1, \mathrm{n}$ has at least one odd prime factor $\mathrm{q}$. We consider cases depending upon the value of $c=n / q$.

Case (i) $c \equiv 3(\bmod 4)$. The first entry in the c-th column is $\left(\begin{array}{c}(c+1) / 4 \\ (c-3) / 4\end{array}\right)=\left(\begin{array}{c}(c+1) / 4 \\ 1\end{array}\right)$. Thus, by Lemma 1, the coefficient 


$$
\left(\begin{array}{c}
q(c+1) / 4 \\
q
\end{array}\right)
$$

must be in the n-th column and so divisible by its row number. However, this coefficient is clearly not divisible by its row number since $q$ is prime. Hence this case cannot occur.

Case (ii) $c \equiv 1(\bmod 12), c>1$. By Lemma 2 the last entry in the $c=(12 t+1)$-th column is $\left(\begin{array}{c}4 t \\ 1\end{array}\right)$. It follows from Lemma 1 that $\left(\begin{array}{c}4 t q \\ q\end{array}\right)$ is in the n-th column, and so divisible by its row number. However, clearly $4 \mathrm{tq} \nmid\left(\begin{array}{c}4 \mathrm{tq} \\ \mathrm{q}\end{array}\right)$ since $q$ is prime. Thus this case cannot occur.

Case (iii) $c \equiv 5(\bmod 12), c>5$. By Lemma 2 the last entry in the $c=(12 t+5)-t h$ column is $\left(\begin{array}{c}4 t+1 \\ 2\end{array}\right)$. Thus, by Lemma 1 ,

$$
\left(\begin{array}{c}
4 t+1 q \\
2 q
\end{array}\right)=\frac{[(4 t+1) q][(4 t+1) q-1] \ldots[(4 t+1) q-q] \ldots[(4 t+1) q-2 q+1]}{2 q(2 q-1) \ldots q \ldots 1}
$$

is in the $n-t h$ column, and so divisible by its row number $(4 t+1) q$. Since the numerator of the fraction in (4.2) apart from $(4 t+1) q$, is divisible by $q$ but not by $\mathrm{q}^{2}$, while the denominator is clearly divisible by $\mathrm{q}^{2}$, we have

$$
(4 t+1) q-q \equiv 0\left(\bmod q^{2}\right),
$$

that is

$$
t \equiv 0(\bmod q) \text {. }
$$

Again by Lemma 2 the next to the last entry in the $c=(12 t+5)-$ th column is $\left(\begin{array}{c}4 t \\ 5\end{array}\right)$ provided $c>17$ (so that the column contains at least two entries). Thus, by Lemma 1 , the binomial coefficient

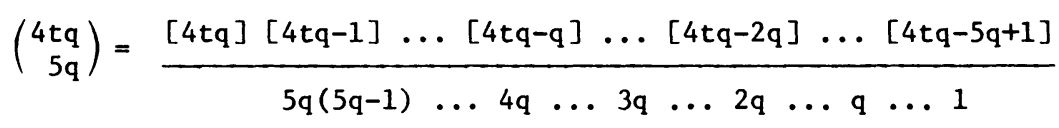

must occur in the n-th column, and so must be divisible by its row number $4 \mathrm{tq}$. The denominator of the fraction in (4.4) is divisible by at least $q^{5}$, while the numerator apart from $4 \mathrm{tq}$, by $(4.3)$, is divisible only by $q^{4}$, if $q>3$, showing that this case is impossible. 
If $q=3$, the $n-t h$ column has the entry $\left(\begin{array}{l}c \\ 0\end{array}\right)=1$ which is clearly not divisible by its row number, so this case is impossible too.

Finally, if $c=17$, the last coefficient in the c-th column is $\left(\begin{array}{l}5 \\ 2\end{array}\right)$, and so, by Lemma $1,\left(\begin{array}{l}5 q \\ 2 q\end{array}\right)$ occurs in the n-th column. By (4.3) this coefficient is not divisible by its row number $5 q$ and so this case is impossible.

Case (iv) $c=5$. We are prevented from using the method of Case (iii) since the fifth column has no entries. We may assume $q \neq 5$ for otherwise $n=25$ and this integer is excluded by the hypothesis of the theorem. Since $q$ is an odd prime, $\mathrm{q}$ is congruent to $3(\bmod 4), 1(\bmod 12)$, or $5(\bmod 12)$. Interchanging the roles of $c$ and $q$, and appealing to the previous cases we see that Case (iv) is impossible too.

Hence $c=1$ and $n$ must be an odd prime.

\section{COEFFICIENTS IN AN EVEN-NUMBERED COLUMN OF A 3-SHIFTED ARRAY}

In a 3-shifted array we are not able to show that only finitely many evennumbered columns have the property that all their entries are divisible by their row numbers. The idea used to treat odd-numbered columns fails, since, for example, with $\mathrm{n}=226$ the last two entries in the 113-th column give rise to (by Lemma 1 ) the coefficients $\left(\begin{array}{l}72 \\ 10\end{array}\right)$ and $\left(\begin{array}{c}74 \\ 4\end{array}\right)$ in the 226-th column, but these are both divisible by their row numbers. However, the 226-th column contains the coefficient $\left(\begin{array}{l}62 \\ 40\end{array}\right)$ which is not divisible by its row number 62 .

THEOREM 3. Let $\mathrm{n}$ be an even composite number 4. Then, if every coefficient in the n-th column is divisible by its row number after a 3-shirt, we must have $\mathrm{n} \equiv 10$ or $34(\bmod 96)$.

PROOF. Assume otherwise. If $\mathrm{n} \equiv 0(\bmod 4)$ we have an immediate contradiction as these columns contain the binomial coefficient $\left(\begin{array}{l}n / 4 \\ n / 4\end{array}\right)=1$, and 1 is certainly not divisible by its row number since $\mathrm{n}>4$. If $\mathrm{n} \equiv 6$ (mod 8 ), say, $\mathrm{n}=8 \mathrm{~d}-2$ the first entry in its column is $\left(\begin{array}{l}2 \mathrm{~d} \\ 2 \mathrm{~d}-2\end{array}\right)=\left(\begin{array}{c}2 \mathrm{~d} \\ 2\end{array}\right) \neq 0(\bmod 2 \mathrm{~d})$, a contradiction. If $n \equiv 0(\bmod 3)$ the $n-t h$ column has the entry $\left(\begin{array}{c}n / 3 \\ 0\end{array}\right)=1$, a contradiction as $n>4$. If $n \equiv 2(\bmod 3)$ the last entry in the $n-t h$ column is $\left(\begin{array}{c}(n-2) / 3 \\ 2\end{array}\right)$. This coefficient is not divisible by its row number since 2 is prime. Thus, we must have $n \equiv 10$ or $34(\bmod 48)$. We have assumes $n \not 10$ or 34 
$(\bmod 96)$ so $\mathrm{n} \equiv 58$ or $82(\bmod 96)$.

If $\mathrm{n} \equiv 58(\bmod 96)$ the first coefficient in the $\mathrm{n}$-th column is

$\left(\begin{array}{c}(n+2) / 4 \\ (n-6) / 4\end{array}\right)=\left(\begin{array}{c}(n+2) / 4 \\ 2\end{array}\right)$. Consequent1y, the second entry in this column is

$\left(\begin{array}{l}(n+6) / 4 \\ (n-18) / 4\end{array}\right)=\left(\begin{array}{c}(n+6) / 4 \\ 6\end{array}\right)$. This coefficient is not divisible by its row number since it is equal to (setting $n=96 \ell+58$ )

$$
(24 \ell+16) \quad\left(\frac{(24 \ell+15)(24 \ell+14)(24 \ell+13)(24 \ell+12)(24 \ell+11)}{6 \cdot 5 \cdot 4 \cdot 3 \cdot 2 \cdot 1}\right)
$$

The denominator in (5.1) is clearly divisible by $2^{4}$ but $(24 \ell+15) \ldots(24 \ell+11)$ is divisible by at most $2^{3}$. The argument is similar if $\mathrm{n} \equiv 82(\bmod 96)$.

This completes the proof of Theorem 3.

\section{TABLES OF VALUES FOR $j=3$.}

In Table 1 the numerical values of the coefficients in the first 34 columns of the 3-shifted array are indicated. In Table 2 the binomial coefficients, from which the values in Table 1 are computed, are given for odd $n \leq 25$.

$\underline{\text { Table } 1}$

\begin{tabular}{|c|c|c|c|c|c|c|c|c|c|c|c|c|c|c|c|c|c|c|c|c|c|c|}
\hline & 0 & 1 & 2 & & 3 & 4 & 5 & & 6 & 7 & & 8 & 9 & & 10 & 11 & 12 & 13 & 14 & 15 & & \\
\hline 0 & 1 & & & & & & & & & & & & & & & & & & & & & \\
\hline 1 & & & & & 1 & 1 & & & & & & & & & & & & & & & & \\
\hline 2 & & & & & & & & & 1 & 2 & & 1 & & & & & & & & & & \\
\hline 3 & & & & & & & & & & & & & 1 & & 3 & 3 & 1 & & & & & \\
\hline 4 & & & & & & & & & & & & & & & & & 1 & 4 & 6 & 4 & & 1 \\
\hline 5 & & & & & & & & & & & & & & & & & & & & 1 & & 5 \\
\hline & 18 & 19 & & 20 & 21 & & 22 & 23 & & 24 & 25 & 26 & & 27 & 28 & 29 & 30 & 31 & 32 & 33 & & 34 \\
\hline 5 & 10 & & 5 & 1 & & & & & & & & & & & & & & & & & & \\
\hline 6 & 1 & & 6 & 15 & 20 & & 15 & 6 & & 1 & & & & & & & & & & & & \\
\hline 7 & & & & & 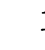 & 1 & 7 & 21 & & 35 & 35 & 2 & & 7 & 1 & & & & & & & \\
\hline 8 & & & & & & & & & & 1 & 8 & 2 & & 56 & 70 & 56 & 28 & 8 & 1 & & & \\
\hline 9 & & & & & & & & & & & & & & 1 & 9 & 36 & 84 & 126 & 126 & 84 & & 36 \\
\hline 10 & & & & & & & & & & & & & & & & & 1 & 10 & 45 & 120 & & 10 \\
\hline 11 & & & & & & & & & & & & & & & & & & & & 1 & & 11 \\
\hline
\end{tabular}


Table 2

\begin{tabular}{|c|c|c|c|c|c|c|c|c|c|c|c|c|c|}
\hline & 1 & 3 & 5 & 7 & 9 & 11 & 13 & 15 & 17 & 19 & 21 & 23 & 25 \\
\hline 1 & & $\left(\begin{array}{l}1 \\
0\end{array}\right)$ & & & & & & & & & & & \\
\hline 2 & & & & $\left(\begin{array}{l}2 \\
1\end{array}\right)$ & & & & & & & & & \\
\hline 3 & & & & & $\left(\begin{array}{l}3 \\
0\end{array}\right)$ & $\left(\begin{array}{l}3 \\
2\end{array}\right)$ & & & & & & & \\
\hline 4 & & & & & & & $\left(\begin{array}{l}4 \\
1\end{array}\right.$ & $\left(\begin{array}{l}4 \\
3\end{array}\right)$ & & & & & \\
\hline 5 & & & & & & & & $\left(\begin{array}{l}5 \\
0\end{array}\right)$ & $\left(\begin{array}{l}5 \\
2\end{array}\right)$ & $\left(\begin{array}{l}5 \\
4\end{array}\right)$ & & & \\
\hline 6 & & & & & & & & & & $\left(\begin{array}{l}6 \\
1\end{array}\right)$ & $\left(\begin{array}{l}6 \\
3\end{array}\right)$ & $\left(\begin{array}{l}6 \\
5\end{array}\right)$ & \\
\hline 7 & & & & & & & & & & & $\left(\begin{array}{l}7 \\
0\end{array}\right)$ & $\left(\begin{array}{l}7 \\
2\end{array}\right)$ & $\left(\begin{array}{l}7 \\
4\end{array}\right.$ \\
\hline 8 & & & & & & & & & & & & & ( \\
\hline
\end{tabular}

\section{ARBITRARY ARITHMETIC PROGRESSIONS}

Let $a k+b$ be any arithmetic progression and let positive integers of the form $a k+b$ be arranged in rows and columns as in (1.2). Consider the "triangular" array obtained from (1.1) by deleting the first column and all rows not of the form $a k+b$. For example, when $a=4, b=3$, one obtains the display (coefficients divisible by their row number are circled),

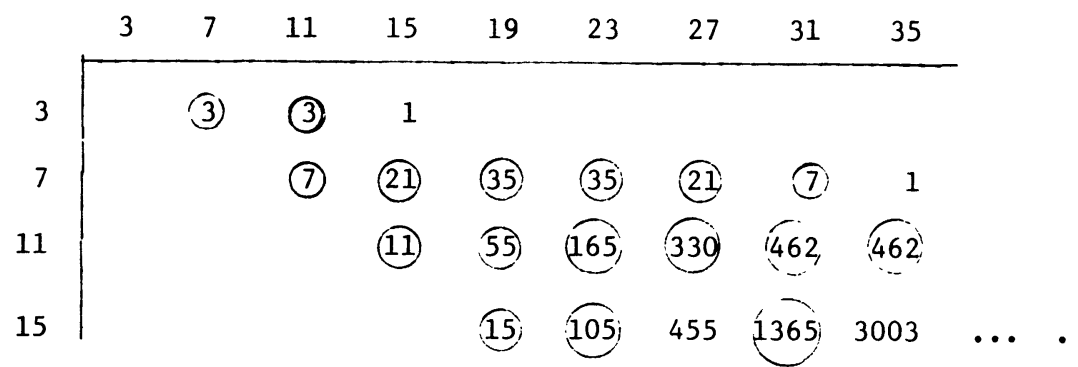

We call such a display an $a k+b$ display, and we investigate, in this section, the proposition that a column number $n$ in an $a k+b$ display is a prime of the form $a k+b$ if and only if every binomial coefficient in its column is divisible by its row number. (The result of Mann and Shanks for such a one-shifted array is the special case $a=1, b=0)$. We obtain this result very simply provided that a does not belong to the finite exceptional set of composite integers with no proper divisor $>a$. 
Let each row after the first in the array (1.1) be shifted one unit to the right after deleting the first column. Let integers of the form $a k+b$ be displayed in rows and columns so that $\left(\begin{array}{l}r \\ s\end{array}\right)$ occurs in the $t-t h$ column, $1 \leq s \leq r$. if and only if $t=r+a s$. Thus, one obtains the $a k+b$ display.

\begin{tabular}{|c|c|c|c|c|c|}
\hline & b & $a+b$ & $2 a+b$ & $3 a+b$ & $4 a+b$ \\
\hline b & & $\left(\begin{array}{l}b \\
1\end{array}\right)$ & $\left(\begin{array}{l}\mathrm{b} \\
2\end{array}\right)$ & $\left(\begin{array}{l}b \\
3\end{array}\right)$ & $\left(\begin{array}{l}b \\
4\end{array}\right)$ \\
\hline$a+b$ & & & $\left(\begin{array}{c}a+b \\
1\end{array}\right)$ & $\left(\begin{array}{c}a+b \\
2\end{array}\right)$ & $\left(\begin{array}{c}a+b \\
3\end{array}\right)$ \\
\hline$+b$ & & & & $\left(\begin{array}{r}2 a+ \\
1\end{array}\right.$ & $\left(\begin{array}{c}2 a+b \\
2\end{array}\right)$ \\
\hline $3 a+b$ & & & & & $\begin{array}{r}3 a+ \\
1\end{array}$ \\
\hline
\end{tabular}

where $\left(\begin{array}{l}r \\ s\end{array}\right)=0$ if $s>r$.

THEOREM 4. Let the integers of the form $a k+b$ be displayed as in (7.1). With the possible exception of the finite set of composite integers which have no proper divisor $>a$, an integer is a prime of the form $a k+b$ if and only if every binomial coefficient in its column is divisible by its row number.

PROOF. The theorem is vacuously true for $n=b$. Let $n=p$ where $p$ is a prime $>\mathrm{b}$ and assume that $\mathrm{r} \nmid\left(\begin{array}{l}\mathrm{r} \\ \mathrm{s}\end{array}\right)$ where $\left(\begin{array}{l}\mathrm{r} \\ \mathrm{s}\end{array}\right)$ is in the p-th column. On one hand, we have

$$
\left(\begin{array}{c}
r-1 \\
s-1
\end{array}\right)(r)=\left(\begin{array}{l}
r \\
s
\end{array}\right)(s),
$$

so that $(r, s)>1$. On the other hand, we have $p=r+a s$ so that $(r, s) \mid p$, a clear contradiction to the primality of $p$.

Let $\mathrm{n}=\mathrm{a} \mathrm{k}+\mathrm{b}$ be a composite integer, say $\mathrm{n}=\mathrm{p}$, where $\mathrm{p}$ is a prime and $l=a j+d, j \geq 1,0 \leq d<a$. Then $\left(\begin{array}{c}p((j-1)(a)+d) \\ p\end{array}\right)$ is in the $n=p l$-th column since $p((j-1)(a)+d)+a p=p(a j+d)$. But $p s \nmid\left(\begin{array}{c}p s \\ p\end{array}\right)$ if $p$ is prime; see, e.g. [2, p. 132]. Thus $n$ has a binomial coefficient in its column which is not divisible by its row number. Q.E.D.

COROLLARY. A positive integer $\mathrm{n}>1$ is prime if and only if every binomial coefficient in its column in the display (7.1) is divisible by its row number.

PROOF. Let $a=1$ and $b=0$ in Theorem 4 . The composite integer $n=1$ is 
exceptional since every coefficient in its column (there are none) is divisible by its row number. Since 1 has no (proper) divisor greater than itself the conditions of Theorem 4 are satisfied. Q.E.D.

REMARK 7.1 When $a=1$ and $b=0$ the display (7.1) appears as

\begin{tabular}{|c|c|c|c|c|c|c|c|c|c|c|c|}
\hline 1 & 2 & 3 & 4 & 5 & 6 & 7 & 8 & 9 & 10 & 11 & 12 \\
\hline 1 & 1 & & & & & & & & & & \\
\hline 2 & & 2 & 1 & & & & & & & & \\
\hline 3 & & & 3 & 3 & 1 & & & & & & \\
\hline 4 & & & & 4 & 6 & 4 & 1 & & & & \\
\hline 5 & & & & & 5 & 10 & 10 & 5 & 1 & & \\
\hline 6 & & & & & & 6 & 15 & 20 & 15 & 6 & 1 \\
\hline
\end{tabular}

REMARK 7.2. The proof of Theorem 4 is, in addition to its generality, even simpler than the proof of the Theorem of Mann and Shanks [2] in that it is not necessary to consider separately primes of the form $6 k+1$ and $6 k-1$.

REMARK 7.3. In Theorem 4, the integer $d$ may or may not be equal to $b$. If not, it is understood that the coefficient $\left(\begin{array}{c}(j-1) a+d \\ 1\end{array}\right)$ occurs in the a $j+d$ column of an a $j+d$ display. This display is not the same as (7.1), but is completely analogous and can be easily obtained by the reader.

REMARK 7.4. The necessary condition proved above is equivalent to the assertion that if $a$ is a prime of form $a k+b$ each coefficient of terms of degree $n$ in $\sum_{k=0}^{\infty}\left(x+x^{2}\right)^{a k+b}-x^{a k+b}$ is divisible by $n$ (see $\left.[2, p .134]\right)$. Some illustrative examples.

Example 1. $a=4, b=1,3$.

Binomial coefficients which are divisible by their row numbers are circled to distinguish them from ordinary binomial coefficients which are enclosed (as is usual) in parentheses. 


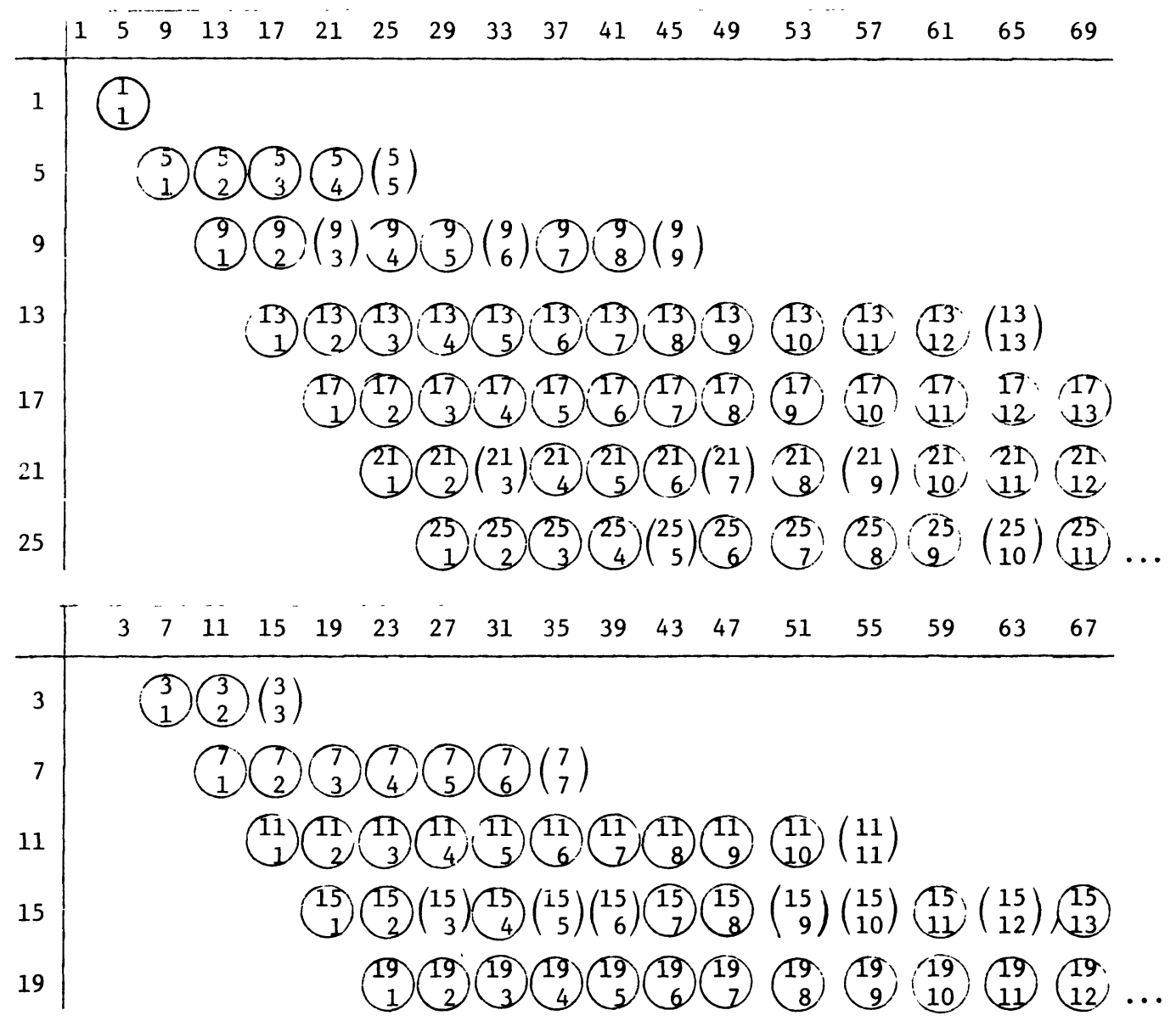

REMARK 7.5. Note that 9 is an exception, as well as 1 , in that it is composite but every coefficient in its column is divisible by its row number. Note that $9=3^{2}$ and $3<4$ so that the exception does not contradict Theorem 4 . There are no other exceptions for the moduius 4 . 
Example 2. $a=7, b=4$ and $a=11, b=7$.

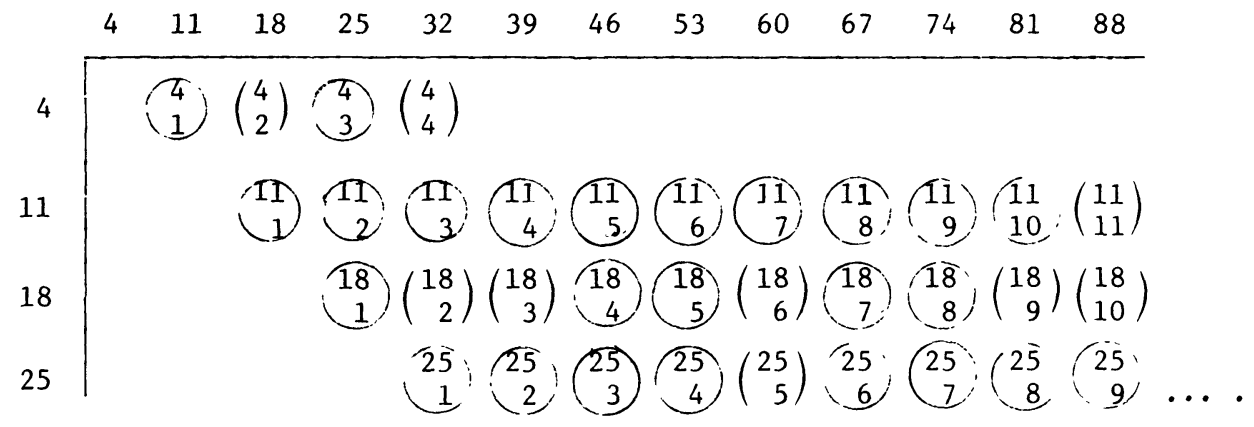

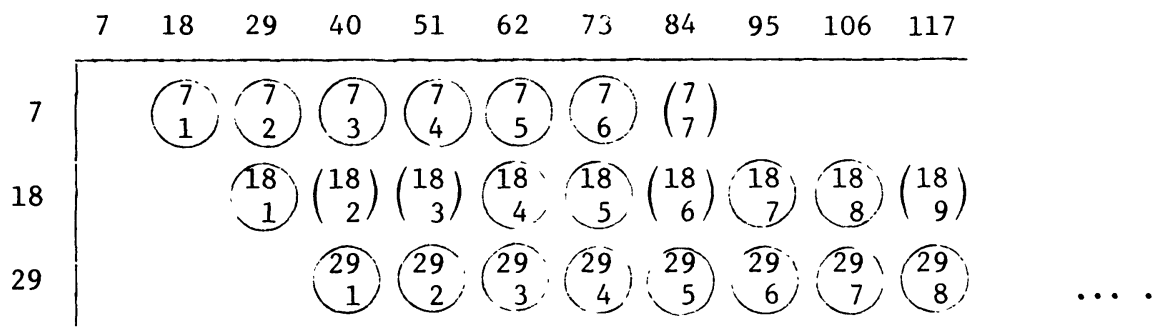

REMARK 7.6. Note that 18 has a proper divisor $>7$ but not greater than 11. The only composite integers with all coefficients in their column divisible by their row number are 4 in the first display (note $4<7$ ) and 18 in the second display. of course, the desired coefficient, guaranteed by Theorem 4 , appears only if the displays are extended. For example, if $n=88=2 \cdot 44$ the coefficient not divisible by its row number (guaranteed by Theorem 4 ) is $\left(\begin{array}{c}74 \\ 2\end{array}\right)$. The coefficient $\left(\begin{array}{c}37 \\ 1\end{array}\right)$ is the first coefficient in the 44-th column in a $7 n+2$ display.

\section{REFERENCES}

1. GOULD, H. W. A new primality criterion of Mann and Shanks and its relation to a theorem of Hermite with extension to Fibonomials, Fib. Quart. 10 (1972), pp. 355-364.

2. MANN, HENRY B. and SHANKS, Daniel. A necessary and sufficient condition for primality and its source, J. Comb. Theory 13 (1972), pp. 131-134.

3 SCHUR, I. Arithmetische Eigenschaften der Potenzsummen einer algebraischen Gleichung, Compositio Math. 4 (1937), pp. 432-444. 


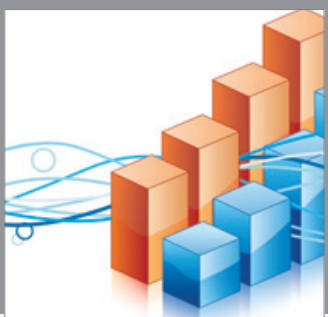

Advances in

Operations Research

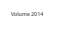

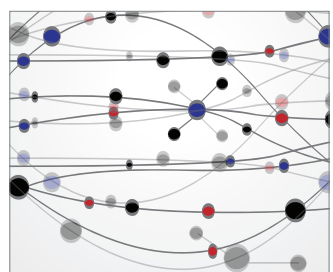

\section{The Scientific} World Journal
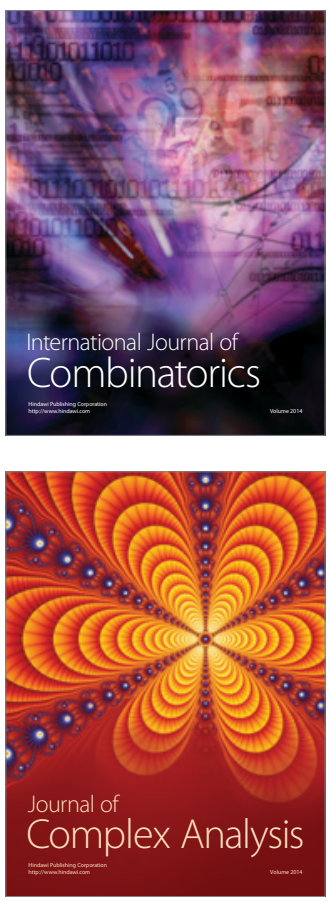

International Journal of

Mathematics and

Mathematical

Sciences
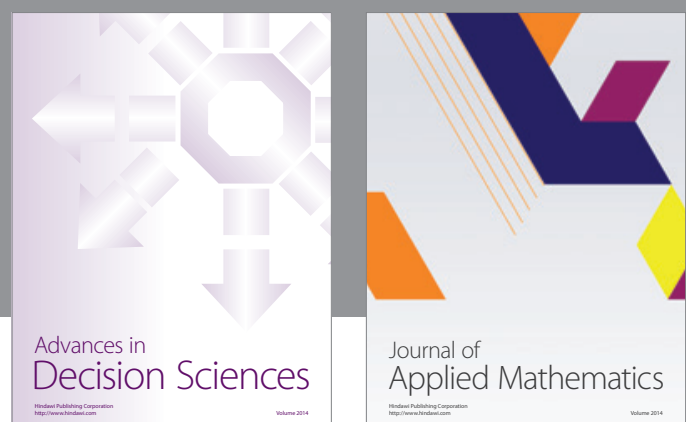

Journal of

Applied Mathematics
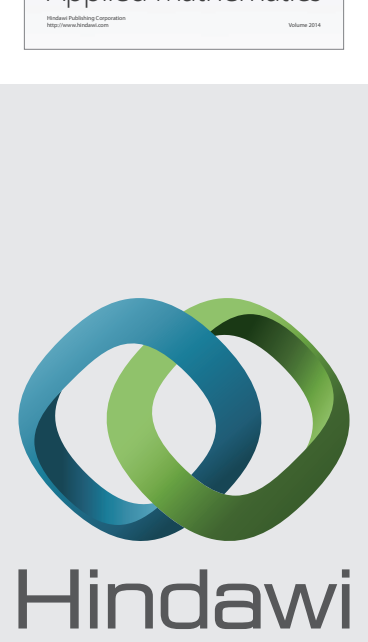

Submit your manuscripts at http://www.hindawi.com
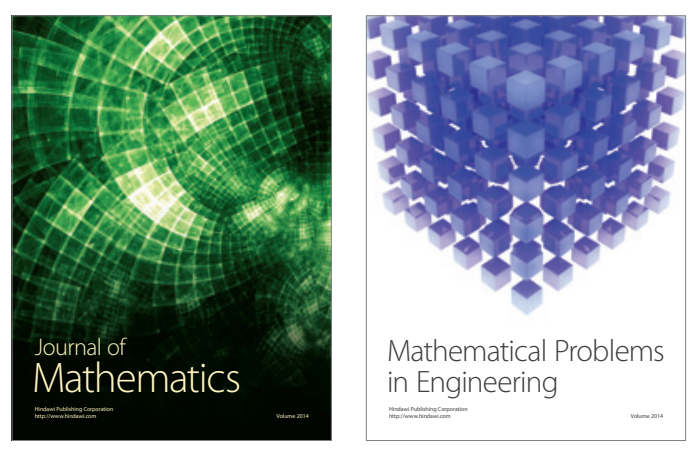

Mathematical Problems in Engineering
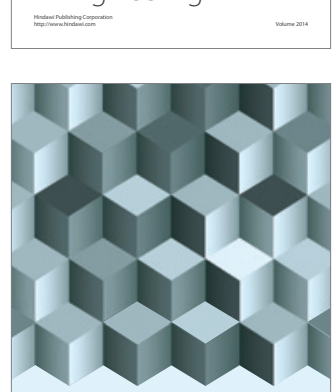

Journal of

Function Spaces
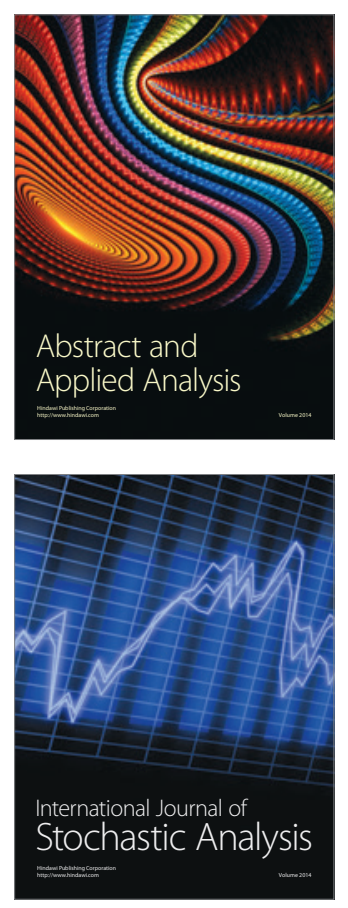

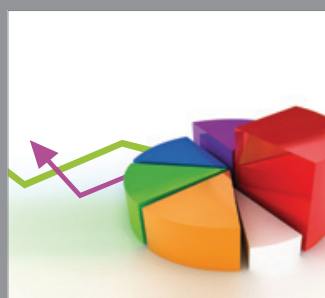

ournal of

Probability and Statistics

Promensencen
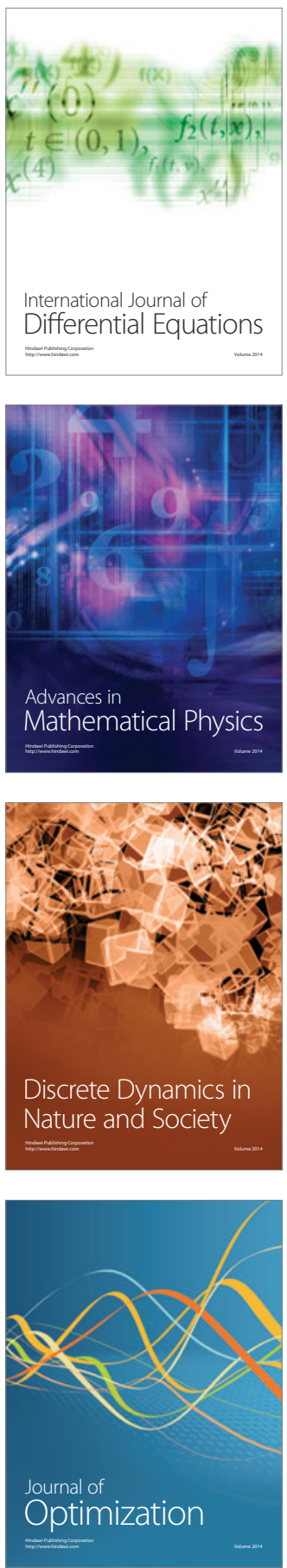